\title{
Form finding of twisted interlaced structures: a hybrid approach
}

\author{
Sina Nabaei \\ Chaire of Timber Construction (IBOIS), École Polytechnique Fédérale de Lausanne \\ (EPFL), CH-1015, Lausanne, Switzerland, sina.nabaei@epfl.ch

\section{Olivier Baverel} \\ Navier Research Unit, École des Ponts ParisTech (ENPC), Champes-Sur-Marne, \\ France, baverel@enpc.fr

\section{Yves Weinand} \\ Chaire of Timber Construction (IBOIS), École Polytechnique Fédérale de Lausanne \\ (EPFL), CH-1015, Lausanne, Switzerland, yves.weinand@epfl.ch
}

\begin{abstract}
Our study presents a set of form-finding procedures to explore curved structures made from interlaced panels. Interlacing introduces a particular coupling between assembly components which has to be formulated along with a pertinent flexible body model. We examine here a hybrid approach: panels are simulated a first time using an elastic rod model formulated within a constrained elastic energy minimization where user can virtually buckle, twist and interlace strip assemblies. A thin shell model dynamically integrated comes complementary to the rod approach in order to resolve intersections in case of panels colliding while interlaced. Some conceptual structures are presented to demonstrated the procedure.
\end{abstract}

\section{Introduction}

Interlacing can be regarded as a unifying process to create an assembly from individual components: e.g. knitting a tissue from yarns, weaving a basket from wood strips. Interlacing, regardless of scale, inherits some elegant structural notions to be brought beyond the wickerwork scale. It lets assemble flexible slender components into a resistant object, with far more load bearing capacity. It lets span over a distance times more than the cross section dimension of its components. Complex coupling introduced by interlacing also distributes forces over the assembly components to ensure a more homogeneous load take down. While interlacing with tensile elements is more matter of pattern complexity, some key differences impose when assembling flexible components with bending resistance and anisotropic cross section. They are flexible enough to undergo large elastic deformations but would 

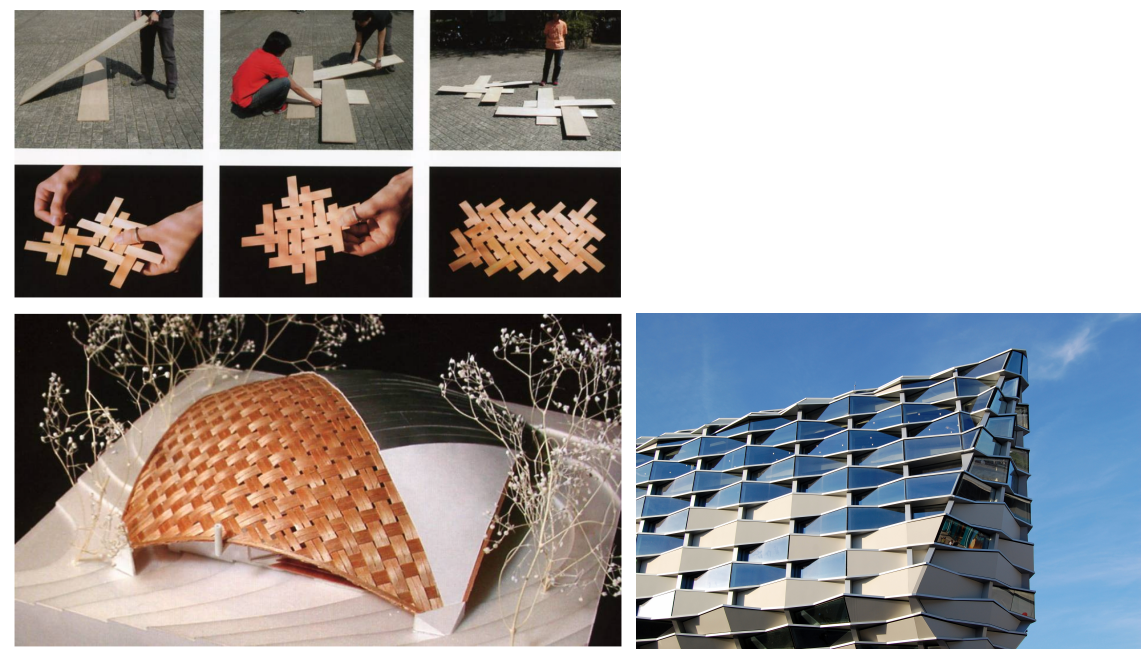

(a) Wickerwork house, Nagano, Japan, 2002, Shigeru (b) Facade of the Pabellón de Aragón, Expo Ban architects 2008, Zaragoza, Spain

Figure 1: Interlacing: structural versus visual influence

not allow any arbitrary pattern unlike textiles: panels would fail in tight closure or high curvature patterns. Bending and twisting forces influence how the interlaced relaxed geometry would look like. The fundamental interest of reproducing a pattern with components resistant in bending and torsion (unlike tensile-only ones) is that the assembly will relax into an actively curved geometry which can span over an architectural ambiance. Curved spanning (versus being a tissue) is the key to a structural regard toward interlaced assemblies rather than a facade motif or an ornament. (see Fig.1) This forward implies that the geometry for an actively curved interlace of components with bending and torsional resistance has to be form-found. This form-finding problem with all implied complexities is explored in this manuscript. We have to adopt a nonlinear structural model to simulate panels with bending and twist degrees of freedom, have to come up with a formulation to impose an interlacing pattern as coupling constraints and perform collision detection/handling if required. In terms of technical details, the Euler Elastica [Love 2013] (see Fig. 2) is the building block of the specific family of structures highlighted in our study. We use an extended energy functional to take into account also for twist contributions and append three further structural design potentials on top of a simple Elastica: we explore twisted Elastica made from flexible panels and couple these individual Elasticas with proximity and/or alignment constraints coming from our desired interlace design pattern. We also deal with collision resolving using a pseudo-dynamic shell solver in case interlace and twist boundary condition cause panel intersection.

The manuscript is organized in following order: the related work is briefly re- 


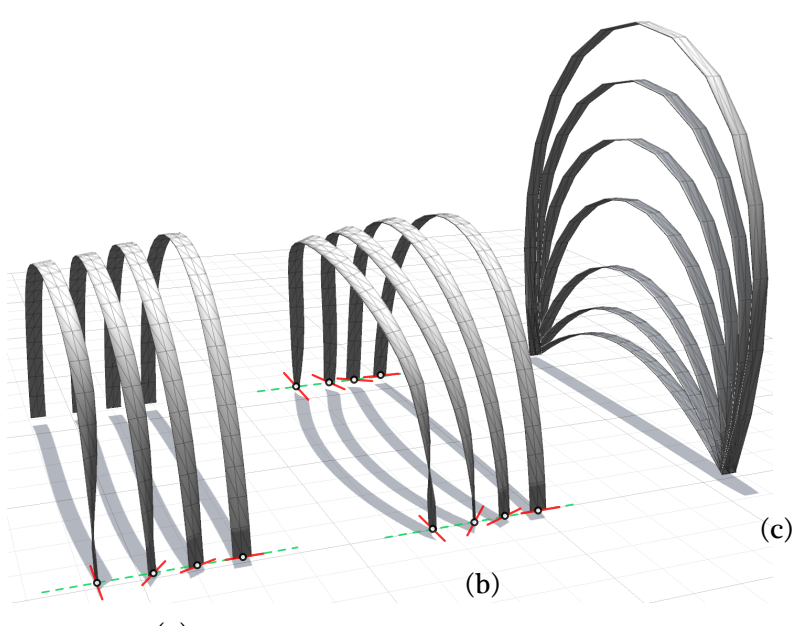

(a)

Figure 2: Elastica variants with and without end twist for timber panel $0.24 m \times 0.015 m$ spanning over $6 \mathrm{~m}$ : (a) twist applied on single end vertex (b) symmetric twist applied on both end vertices (c) the zero twist Elastica solutions for various total lengths from $6.5 \mathrm{~m}$ up to $16 \mathrm{~m}$

viewed spanning over form-active structures, physics-based rod and shell models. Our hybrid form-finding approach is then detailed, rod-wise and shell-wise and is put in practice for some case-study concept structures to demonstrate the design potential of the procedure.

\subsection{Related work}

Interlaced/form-active structures still most of applications for form-active structures made from timber panels come from research institutes, hereby four among the recent pavilions are cited: the wood pavilion of Oslo School of Architecture and Design is a modular structural web, made out of thin pine veneer naturally curved and post-tensioned by material's hygroscopic effect [Hensel 2013]. (Fig. 3(a)) ICD/ITKE research pavilion [Fleischmann and Menges 2012] (Fig. 3(b)) highlights an overall interlaced geometry using actively-bent timber panels connected by clip dovetail joints and clamped at both ends. The "Radical Wood Pavilion" of Design + Analysis research group of Aalto University [Niiranen 2013] is a form-active, interlaced structure, highlighting a twisted module. (Fig. 3(c)) Timber Fabric structures of IBOIS-EPFL, Switzerland [Hudert 2012] is about interpretation of knotted and braided patterns using flexible timber panels as strands. Panels are elastically bent and twisted into a curved arch structure (Fig. 3(d)) where the geometry comes from the assembly logic.

Elastic rods Elastic rods have been topic of intense ongoing research. Covered subjects go from computational mechanics [Dias and Audoly 2014], DNA super- 


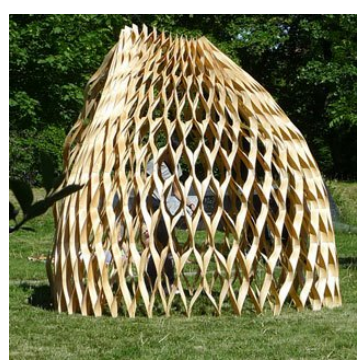

(a)

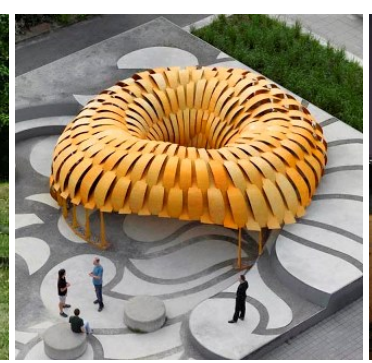

(b)

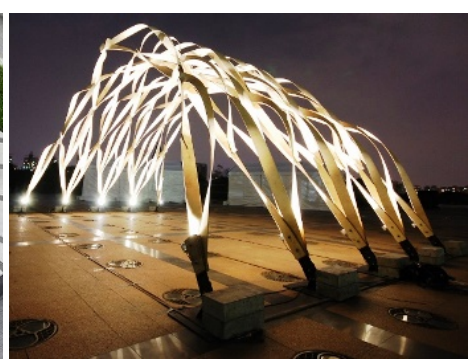

(c)

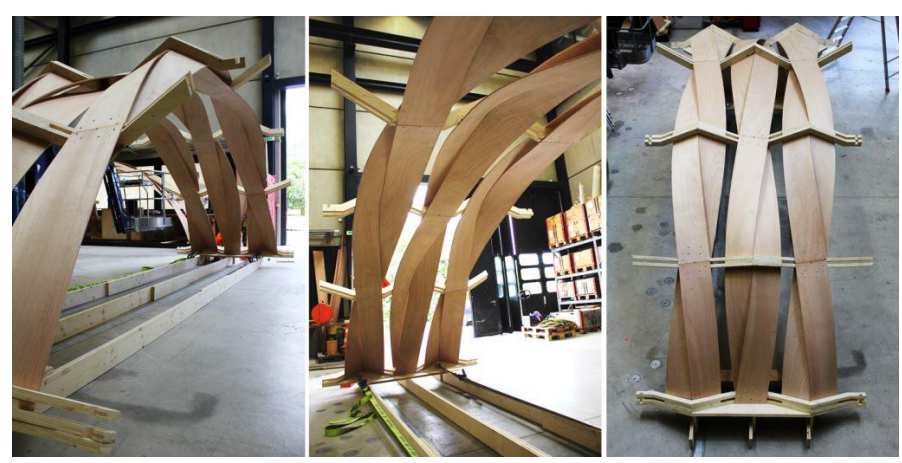

(d)

Figure 3: Interlaced form-active structures made form flexible panels: (a) The wood Pavilion of Oslo School of Architecture and Design, (b) ICD/ITKE research pavilion 2010, (c) The "Radical Wood Pavilion" (d) Timber Fabric Structures: photo (c) Markus Hudert

coiling Simulation [Olson et al. 2013] and robotics [Lock et al. 2010] up to the physically based computer animation and virtual surgery simulators. [Chentanez et al. 2009; Miller et al. 2014]

Thin shell physics-based models Thin shell deformable modeling has been intensively researched through diverse disciplines. Mass spring systems [Choi and Ko 2005] have been among most trivial approaches for physically simulation of cloth and deformable skins while [Bridson et al. 2003; Grinspun et al. 2003] propose a dihedral angle difference as a measure to define the thin shell out of plane behavior. An alternative approach based on discrete isometric bending model is also presented in [Bergou et al. 2006]. Most recently Iso Geometric analysis has also been applied to simulate cloth deformation. [Lu and Zheng 2014]

\subsection{Goals and Contributions}

Our main motivation through this study is thus to formulate a physics-based tool for interlaced configurations to enable an interactive design experience. 
Rich rod and shell models for structural architecture applications: we offer implementations of two efficient physics-based models from the computer graphics body of knowledge [Bergou et al. 2008; Grinspun et al. 2003] to use for architectural geometry applications. The fact of using these models bring some key features into the existing particle-based form-finding tools such as [Piker 2013]: (i) the possibility to deal with general rod and shell cross sections with usual elastic stiffness terms, instead of simplified spring stiffness. Spring values usually need interpretation, depends on the spatial discretization and require special care to create out-of-plane resistance for shell simulations (ii) enhancing twist degrees of freedom for rod deformation (iii) an improved out-of-plane bending behavior for thin shell physically based simulation.

Constraint formulation of an interlaced design pattern: we give elements on how to interpret a desired interlacing pattern as equality constraints for a nonlinear optimization problem. Our formulation integrates also some useful architectural local connection features such as imposing a desired offset distance or the possibility to impose the alignment of panels at the overlap.

Discrete Elastic rod model employed in a static framework: we reformulate the initially proposed dynamically integrated rod model of [Bergou et al. 2008] into an offline constrained optimization problem with displacements and twists as variables of the optimization problem defined all at once.

\section{2 twisted interlaced structure simulation pipeline}

The complexity of interlaced configuration form-finding can be reduced by breaking it down into pertinent sub-problems based on the flexible body model used to represent panels and the physical phenomenon to simulate. Buckling is the key curving agent in these form-active structures and supposing a static framework, it can be readily formulated as a constrained elastic energy minimization with the only constraints as total panel length and span. A flexible rod model with vertex positions and cross section orientation of edge segments as variables, can be efficiently used to get elastic energy expressions. Having end vertices twisted and panels which have to pass underneath or above of another panel of the assembly at a particular position (interlacing) are simply additional constraints on either rod vertices or cross section orientation angles (twists), which have to be injected to the initial elastic energy minimization problem of buckling. The notion of above and below have to be discussed (is it with respect to global vertical axis or the local one?) as well as the clearance at overlap nodes, the minimum required offset. Up to here if the minimum elastic energy geometry does not contain colliding panels, all of above mentioned can be solved using constrained optimization formulated with a rod kinematics. Collision detection/resolving in its turn seems to us easier to handle with a shell kinematics in a dynamic simulation, which motivates our hybrid approach. This brief go-through also provides indications on the inputs to be specified for each problem, the design parameters. For each panel, the initial length and target span, the imposed end twist 
and material properties have to be specified by user. At each overlapped node the offset distance to respect, is also an input data. These design parameters are symbolically represented on Fig. 4 at problem definition level. While investigating an interlaced configuration, it is also convenient to represent and discuss the pattern in a connectivity graph. The interlaced diagram is introduced for this purpose. Since the location of overlap node is also a degree of freedom for our problem, user has also to specify where (on which rod vertices) he would like to create the overlap coupling and his desired element order at this particular overlap.

We elaborate the rest of this section as follows: the interlaced diagram is first introduced and technical details for the elastic rod model used to simulate the twisted Elasticas and for the thin-shell model used for intersection resolving are presented briefly afterward. We show how to configure the optimization problems to solve for the classical planar Elastica (without end twists), for the twisted one and for the coupled system of twisted Elasticas with overlap order constraints. The thin-shell model is presented next, followed by our intersection resolving implementation.

\subsection{Interlaced diagram}

A graph representation inspired form projected knot diagrams is introduced in order to define and distinguish interlaced morphologies. Lines numbered at both ends represent panels and for intermediate interlace nodes, the relative overlap order of panels is noted using the $>$ notation. The graph representation enables to effectively sweep over interlaced configurations in an abstract topological form regardless of the dual 3D shape while there might be several of those duals corresponding to the same graph depending on panel's initial lengths and span, their elastic properties, precise location of overlaps and also the crossing's linking number.

\subsection{Static simulation with elastic Kirchhoff rod model}

We assume the panel(s) to be represented by their centerline curve, have a cross section of $w \times t$, a flat initial state (zero initial curvature, zero initial twist) of length $l_{\text {init }}$ and initial orientation defined by vector $\mathbf{n}_{0}$. Our goal is to solve the buckled twisted state of the panel when it spans over $l_{\operatorname{tar}}$ and end twists of $\theta^{0}, \theta^{n}$ are applied on its end vertices, giving the new cross section orientation of end vertices as $\mathbf{n}_{1}$ and $\mathbf{n}_{2}$. (see Fig. 4 top) We consider the energy functional for elastic Kirchhoff rod proposed by [Bergou et al. 2008] and reproduced in Eq. 1 for a rod with $n$ linear edge segments. Basically the smooth centerline curve of the rod is divided into a polyline with linear edge segments. We follow the same notation for scalar and vector quantities as [Bergou et al. 2008]. in Eq. 1, over-lined terms correspond to the quantities at their reference state, $\omega_{i}^{j}$ measures the contribution of bi-normal curvature vector of vertex $i$ on edge segment $j$ and $m_{k}$ takes the relative twist of vertex $k$ into account. We refer interested readers to [Bergou et al. 2008] for further details on the curve-angle model. The following stiffness constants are used for rectangular cross section of panel $w \times t$ with usual notation for elastic constants: the bending stiffness $\mathbf{B}=\left(\begin{array}{cc}E t w^{3} / 12 & 0 \\ 0 & E w t^{3} / 12\end{array}\right)$ and the torsional stiffness $\beta=\frac{G w t^{3}}{3}$ where 


\section{Problem definition}

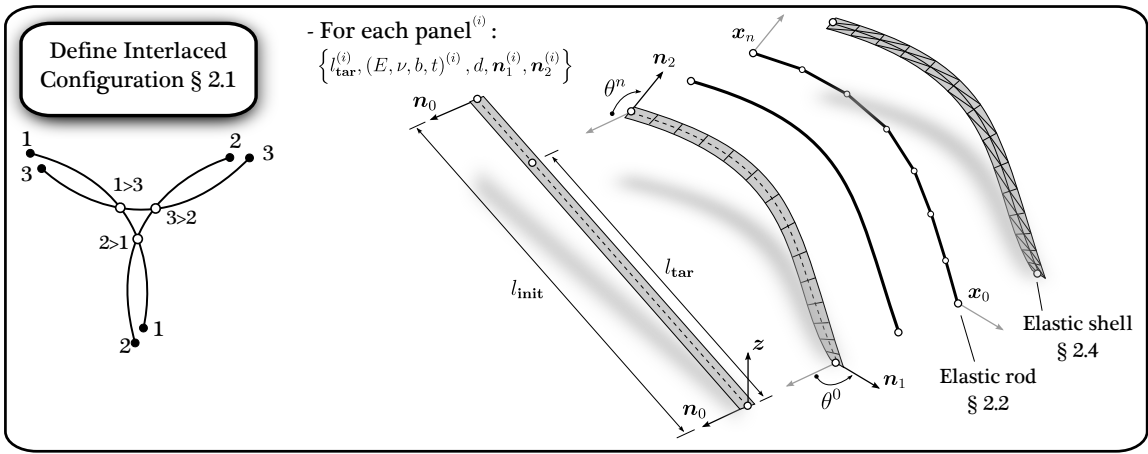

\section{Simulation}

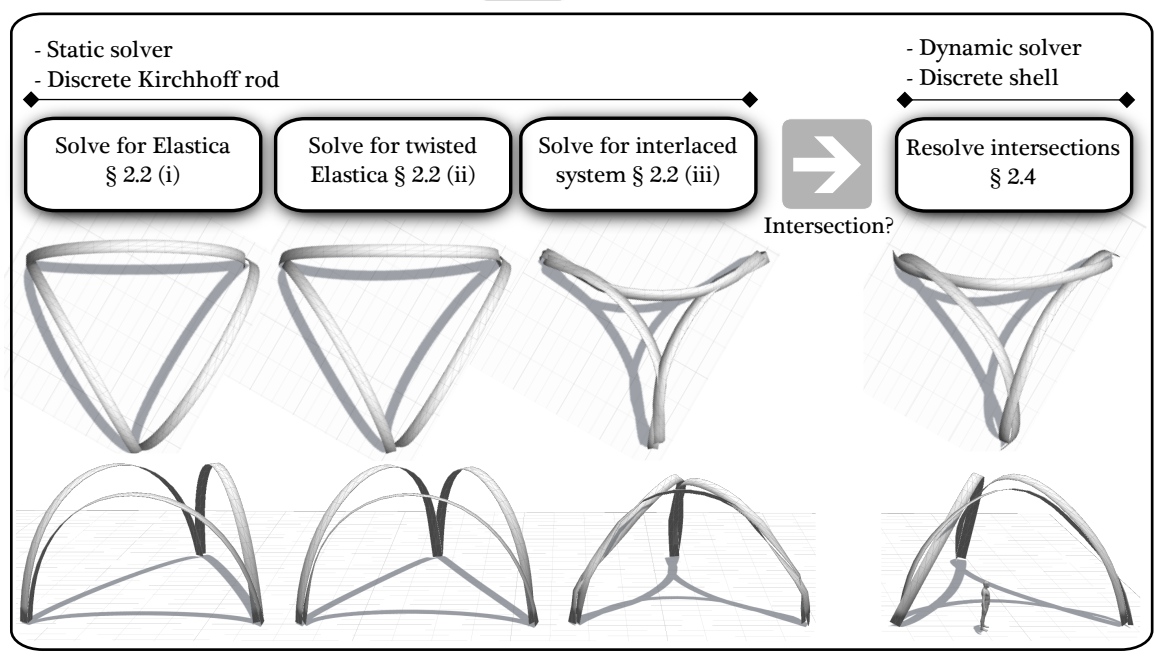

Figure 4: Twisted interlaced structure form-finding pipeline

$G=\frac{E}{2(1+v)}$ is the material shear modulus.

$$
E_{\text {bend }}(\Gamma)=\sum_{i=1}^{n-1} \frac{1}{2 \bar{l}_{i}}\left(\sum_{j=i-1}^{i}\left(\omega_{i}^{j}-\bar{\omega}_{i}^{j}\right)^{T} \mathbf{B}\left(\omega_{i}^{j}-\bar{\omega}_{i}^{j}\right)\right) \quad E_{t w i s t}(\Gamma)=\sum_{k=1}^{n-1} \frac{\beta\left(m_{k}-\bar{m}_{k}\right)^{2}}{\bar{l}_{k}}
$$

The above mentioned energies remains the same for a general cross section, only the stiffness terms are required to be updated. [Bergou et al. 2008] also gives simplified expressions for a rod with an isotropic cross section and deformed from a flat initial state. The original method in [Bergou et al. 2008] is a dynamic simulation and at each time step, before integrating for positions, a quasi-static framework for twist angles has to be satisfied. The inextensibility of edge segments and is particularly 
imposed using the manifold projection method. [Hairer et al. 2006] While the proposed curve-angle kinematic fits well to our problematics, we only require the final relaxed deformed state and thus decide to proceed instead with a static framework for all degrees of freedom and propose to reformulate it as a constrained optimization problem. The coupled system of twisted Elastica is supposed to have of a given number of rods, with $i$ th panel noted as $\Gamma^{(i)}$. Instead of imposing all constraints at once to the flat initial state to get the buckled - twisted - interlaced configuration, we instead proceed with a three stage solution procedure formulated as constrained optimization problems detailed bellow.

I. System of planar Elasticas of imposed total length and span The initial optimization is to solve the Elastica problem with zero twist contribution, which turns out to be the classical bi-hinged Elastica curve. Panel ${ }^{(i)}$ with initial length of $l_{\text {init }}^{(i)}$ buckles to $\operatorname{span} l_{\operatorname{tar}}^{(i)}$, with $n^{(i)}$ equal edges, fixed end vertices and under an additional constraint to keep the deformed configuration in the initial vertical plane. Variables to solve for are unfixed vertex positions (vector quantity $\mathbf{x}$ ) and edge segment twist angle (scalar quantity $\theta$ ). The initial state for this first stage is the flat panel state. (zero initial curvature, zero initial twist)

$$
\begin{aligned}
\min _{\left\{\theta^{(i)}, \mathbf{x}^{(i)}\right\}} & E(\theta, \mathbf{x})=\sum_{(i)=0}^{\Gamma \text { count }} E_{\text {bend }}\left(\Gamma^{(i)}\right)+E_{\text {twist }}\left(\Gamma^{(i)}\right) \\
\text { s.t. } & \text { i) }\left\|\mathbf{x}_{j+1}^{(i)}-\mathbf{x}_{j}^{(i)}\right\|^{2}=\left(l_{\text {init }}^{(i)}\right)^{2} /\left(n^{(i)}\right)^{2}: \quad j \in\left\{0^{(i)}, \ldots, n^{(i)}-1\right\} \\
& \text { ii) } \mathbf{x}_{j}^{(i)}=\overline{\mathbf{x}}_{j}^{(i)}: \quad j=0^{(i)}, n^{(i)} \\
& \text { iii) } \mathbf{n}_{0}^{(i)} \cdot\left(\mathbf{x}_{1}^{(i)}-\mathbf{x}_{0}^{(i)}\right)=0 \\
& \text { iv) } \mathbf{n}_{0}^{(i)} \cdot\left(\mathbf{x}_{n^{(i)}-1}^{(i)}-\mathbf{x}_{n^{(i)}}^{(i)}\right)=0
\end{aligned}
$$

II. System of twisted Elasticas: as the next step we solve for the twisted Elasticas with both end edges supposed to be clamped. The initial state for this analysis are deformed Elastica retrieved by solving problem I described above. The cross section orientation at both ends for each panel are obtained by rotating the corresponding initial normal vector $\mathbf{n}_{0}^{(i)}$ respectively by $\theta^{0^{(i)}}$ and $\theta^{n(i)}$.

$$
\begin{aligned}
& \min _{\left\{\theta^{(i)}, \mathbf{x}^{(i)}\right\}} E(\theta, \mathbf{x})=\sum_{(i)=0}^{\Gamma \text { count }} E_{\text {bend }}\left(\Gamma^{(i)}\right)+E_{t w i s t}\left(\Gamma^{(i)}\right) \\
& \text { s.t. } \text { i) }\left\|\mathbf{x}_{j+1}^{(i)}-\mathbf{x}_{j}^{(i)}\right\|^{2}=\left(l_{\text {init }}^{(i)}\right)^{2} /\left(n^{(i)}\right)^{2}: \quad j \in\left\{1^{(i)}, \ldots, n^{(i)}-2\right\} \\
& \text { ii) } \mathbf{x}_{j}^{(i)}=\overline{\mathbf{x}}_{j}^{(i)}: \quad j=0^{(i)}, 1^{(i)}, n-1^{(i)}, n^{(i)} \\
& \text { iii) Twist angle of material frames for first and last edges of panel }{ }^{(i)} \\
& \text { respectively }\left(\theta^{0}\right)^{(i)},\left(\theta^{n^{(i)}}-1\right)^{(i)} \text { aligned w.r.t } \mathbf{n}_{1}^{(i)} \text { and } \mathbf{n}_{2}^{(i)}
\end{aligned}
$$

III. Interlacing twisted Elasticas: The final simulation step is dedicated to couple twisted panels according to the schematic interlace pattern. The initial state for this 
analysis is the result of problem II. For each overlap panel ${ }^{(i)}>$ panel $^{(k)}$, a handle reference point have to be defined by the user to determine where exactly the overlap has to be passed. The vertices of the panels involved in the overlap (the ones closest to this handle point), noted as $\mathbf{x}_{j}^{(i)}$ and $\mathbf{x}_{l}^{(k)}$, will be the ones to hold the constraints. The distance between corresponding nodes of the overlapping panels, $\left\|\mathbf{x}_{j}^{(i)}-\mathbf{x}_{l}^{(k)}\right\|$, is set to be equal to the given offset distance $d$ and the top/bottom in the overlap is imposed using the projected distance of the involved nodes with respect to the material frame vector $\mathbf{m}_{2}^{(i)}$ of the panel ${ }^{(i)}$. (The out of plane direction of the panel supposed to pass on top of the other one) The former axis gives a local order depending on panel orientation. The global $z$ axis should be used instead of $\left(\mathbf{m}_{2}^{j}\right)^{(i)}$ for a vertically oriented overlap.

$$
\begin{array}{cl}
\min _{\left\{\theta^{(i)}, \mathbf{x}^{(i)}\right\}} & E(\theta, \mathbf{x})=\sum_{(i)=0}^{\Gamma \text { count }} E_{\text {bend }}\left(\Gamma^{(i)}\right)+E_{t w i s t}\left(\Gamma^{(i)}\right) \\
\text { s.t. } & \text { i) }\left\|\mathbf{x}_{j+1}^{(i)}-\mathbf{x}_{j}^{(i)}\right\|^{2}-\left(l_{\text {init }}^{(i)} / n^{(i)}\right)^{2}=0: \quad j \in\left\{1^{(i)}, \ldots, n^{(i)}-2\right\} \\
& \text { ii) } \mathbf{x}_{j}^{(i)}-\overline{\mathbf{x}}_{j}^{(i)}=0: \quad j=0^{(i)}, 1^{(i)}, n-1^{(i)}, n^{(i)} \\
\text { iii) Twist angle of material frames for first and last edges of panel } l^{(i)} \\
\text { respectively }\left(\theta^{0}\right)^{(i)},\left(\theta^{n^{(i)}}-1\right)^{(i)} \text { aligned w.r.t } \mathbf{n}_{1}^{(i)} \text { and } \mathbf{n}_{2}^{(i)} \\
\text { iv) foreach }\left(\mathbf{x}_{j}^{(i)}>\mathbf{x}_{l}^{(k)} \text { order constraint }\right) \\
\left\|\mathbf{x}_{j}^{(i)}-\mathbf{x}_{l}^{(k)}\right\|^{2}-d^{2}=0 \\
\quad\left(\mathbf{x}_{j}^{(i)}-\mathbf{x}_{l}^{(k)}\right) \cdot\left(\mathbf{m}_{2}^{j}\right)^{(i)}-d=0 \\
\text { end foreach }
\end{array}
$$

The above cited problems are solved using a Quasi-Newton procedure with BFGS approximation of the Hessians using a primal-dual interior-point algorithm with a filter line-search method [Wächter and Biegler 2006; HSL]. Notice that only energy gradients with respect to the vertex positions and twist angles are required. (and not the Hessians, see [Bergou et al. 2008] for forces)

\subsection{Pseudo-dynamic simulation with elastic shell model}

In some interlaced configurations with repeatedly inversed overlaps (like overlaps in a braid $\{1>2,2>1,1>2 \ldots\})$ or when the overlap offset distance is small with respect to the panel width, the minimum energy configuration obtained from the coupled Elastica optimization will not be intersection free. We propose to dynamically integrate an elastic shell model of the discretized mid-surface of panels for intersection detection and resolving. Among existing physically based thin shell model we use [Grinspun et al. 2003] for triangle meshes with flexural and membrane energy functionals in their discrete form. The flexural energy comes form a discrete analogy of the integral mean curvature of the surface mesh and is measured as a function of the difference between complements of the dihedral angle of mesh edges on deformed and reference state, the former quantities are marked with an 


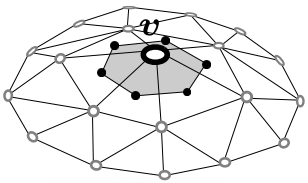

(a)

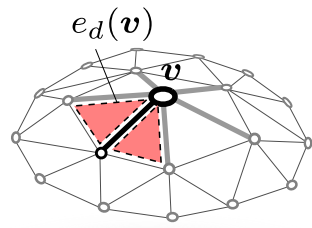

(c)

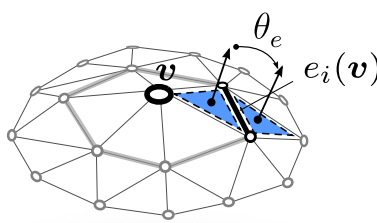

$E_{B}=k_{B} \sum_{\boldsymbol{v}} \sum_{e_{i}(\boldsymbol{v})} \frac{\left(\theta_{e}-\bar{\theta}_{e}\right)^{2}\left\|\bar{e}_{i}\right\|}{2 \bar{h}_{e_{i}}}$

(b)

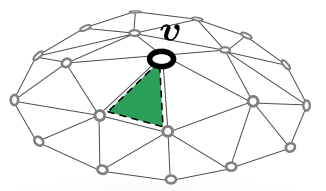

$E_{A}=k_{A} \sum_{f}\left(1-\frac{\|A\|}{\|\bar{A}\|}\right)^{2}\|A\|$

(d)

Figure 5: Discrete elastic shell setting and energies, contributions acting on vertex v: (a) The area (mass) related to vertex (b) Flexural energy contributions of indirect dihedrals around vertex (c) membrane energy contributions of direct dihedrals around vertex (d) in plane shear energy contributions of faces connected to vertex

over line. Principals of discrete elastic shell settings and energies are briefly reminded in Fig. 5. Around a given vertex $\mathbf{v}$ we identify a set of direct dihedrals and corresponding edges noted as $e_{d}(\mathbf{v})$ contributing to membrane forces (in plane tension or compression) and a set of indirect dihedrals and corresponding edges, noted as $e_{i}(\mathbf{v})$ which apply the flexural forces according to the stiff hinge effect around the corresponding edge $e_{i}$. The term $\bar{h}_{e_{i}}$ is the one third of the sum of the height of the two triangles sharing the edge $e_{i}(\mathbf{v})$ at the reference configuration. See also the index of [Grinspun et al. 2003] for another possible definition for $\bar{h}_{e_{i}}$. We apply corresponding values of $k_{L}, k_{A}, k_{B}$ and derivate these energies to get the forces acting on vertices through vector calculus manipulation which we do not reproduce here for conciseness. ([Grinspun et al. 2003] also proposes automatic derivation) the Newmark integration scheme is used for position update with $\gamma=0.5, \beta=0.25$ with the predictor-corrector approach. [Hughes et al. 1979] Mesh intersection detection is performed using the fast triangle-triangle intersection test [Möller 1997] and resolved by applying the corrective penalty forces in direction which minimizes the intersection line as detailed in [Volino and Magnenat-Thalmann 2006]. This method is particularly adopted to our problematic because it does not necessarily 


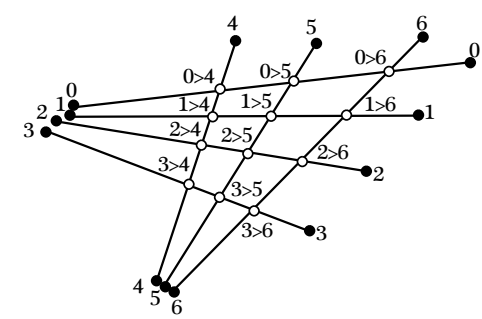

(a)

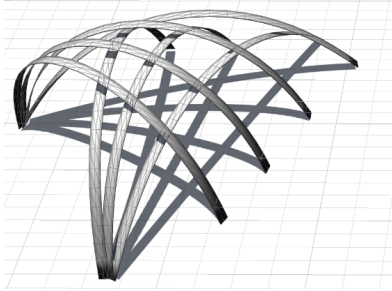

(b)

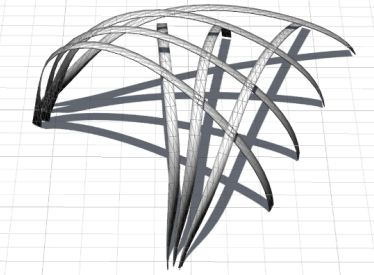

(c)

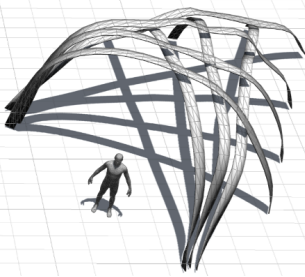

(d)

Figure 6: A gridshell structure with twisted panels: (a) The graph representation (b) the solution to Elastica problem (c) Twisted Elastica configuration (d) Interlaced configuration

require the mesh intersection to be a closed contour (unlike [Baraff et al. 2003]) and moreover, it is history-free.

\section{Case studies}

Four case studies are discussed in this section to demonstrate our form-finding procedure: a gridshell like structure built with twisted panels, a trefoil arch, a pentafoil roof and a twisted truss-beam module. In all these examples panels are supposed to be made from timber and mean elastic properties of wood $E=8000 \mathrm{Mpa}, \boldsymbol{\nu}=$ $0.3, \rho=500 \mathrm{~kg} / \mathrm{m}^{3}$ are used.

\subsection{A gridshell structure with panels}

We provide an example of a gridshell type structure (see Fig.6) this time built with panels instead of regular circular cross-section beams and we additionally impose a uniform twist of $90^{\circ}$ on start vertex of all panels. The beams are all $10 \mathrm{~m}$ long buckled to span various lengths: $6.25 \mathrm{~m}<l<8.46 \mathrm{~m}$. The offset distance is $0.2 \mathrm{~m}$ and alignment of panels is imposed at overlaps. Panels are all $0.24 \mathrm{~m} \times 0.015 \mathrm{~m}$. The structure is at most $3.2 \mathrm{~m}$ high at the level of entrance.

\subsection{An interlaced trefoil}

The concept structure illustrated in Fig.7 is an example of interlacing which requires an intersection handling step. The trefoil arch structure is supposed to be made with timber panels $0.24 m \times 0.015 m$, all panels are $14 m$ long spanning over $8.5 m$ and 


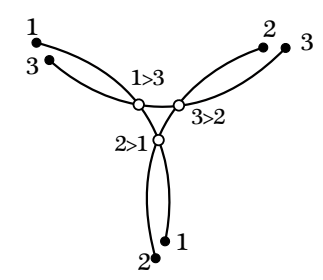

(a)

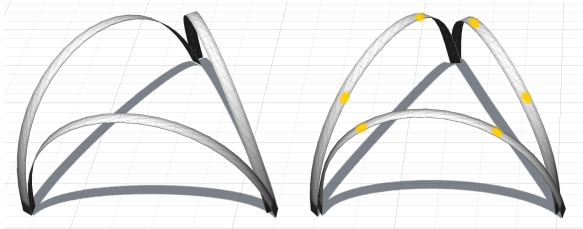

(b) (c)

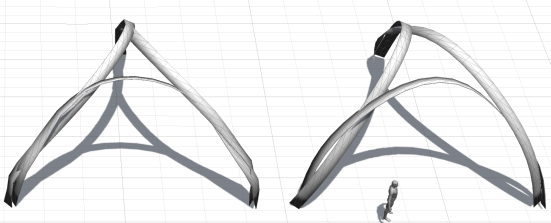

(e)

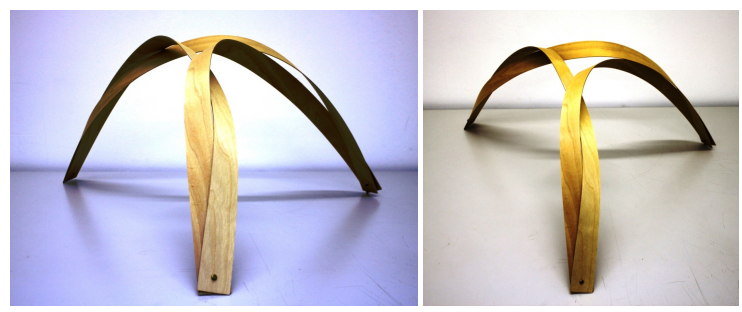

Figure 7: A trefoil interlaced arch: (a) The graph representation (b) the solution to Elastica problem (c) Twisted Elastica configuration with overlapping vertices highlighted (d) Interlaced configuration (contains intersection) (e) Intersections handled

respective twist of $\left(-15^{\circ},-30^{\circ}\right)$ is applied on start and end vertices of each panel. The arch becomes $4.8 \mathrm{~m}$ high at middle-vertex at its intersection-free relaxed state.

\subsection{An interlaced pentafoil}

The concept structure illustrated in Fig. 8 is a complex structure with multiple overlap nodes and colliding components. The pentafoil roof structure is supposed to be made with timber panels $0.65 m \times 0.03 m$, all $14 m$ long spanning over $11.8 m$ with support points equally spaced on a circle of $6.3 \mathrm{~m}$ radius. Respective twist of $\left(34^{\circ}, 34^{\circ}\right)$ is applied on start and end vertices of each panel. As a result of interlacing the arch lifts up to clear $1.98 \mathrm{~m}$ vertically with respect to the support point at its intersection-free relaxed state.

\subsection{Twisted truss module}

Fig. 9 demonstrates an example where the coupling constraint is only a proximity one and no alignment is imposed. Exterior beams are $6.8 \mathrm{~m}$ long and interior ones $6.3 m$ and the span is $6 m$. The width of the beam (axis to axis between exterior 


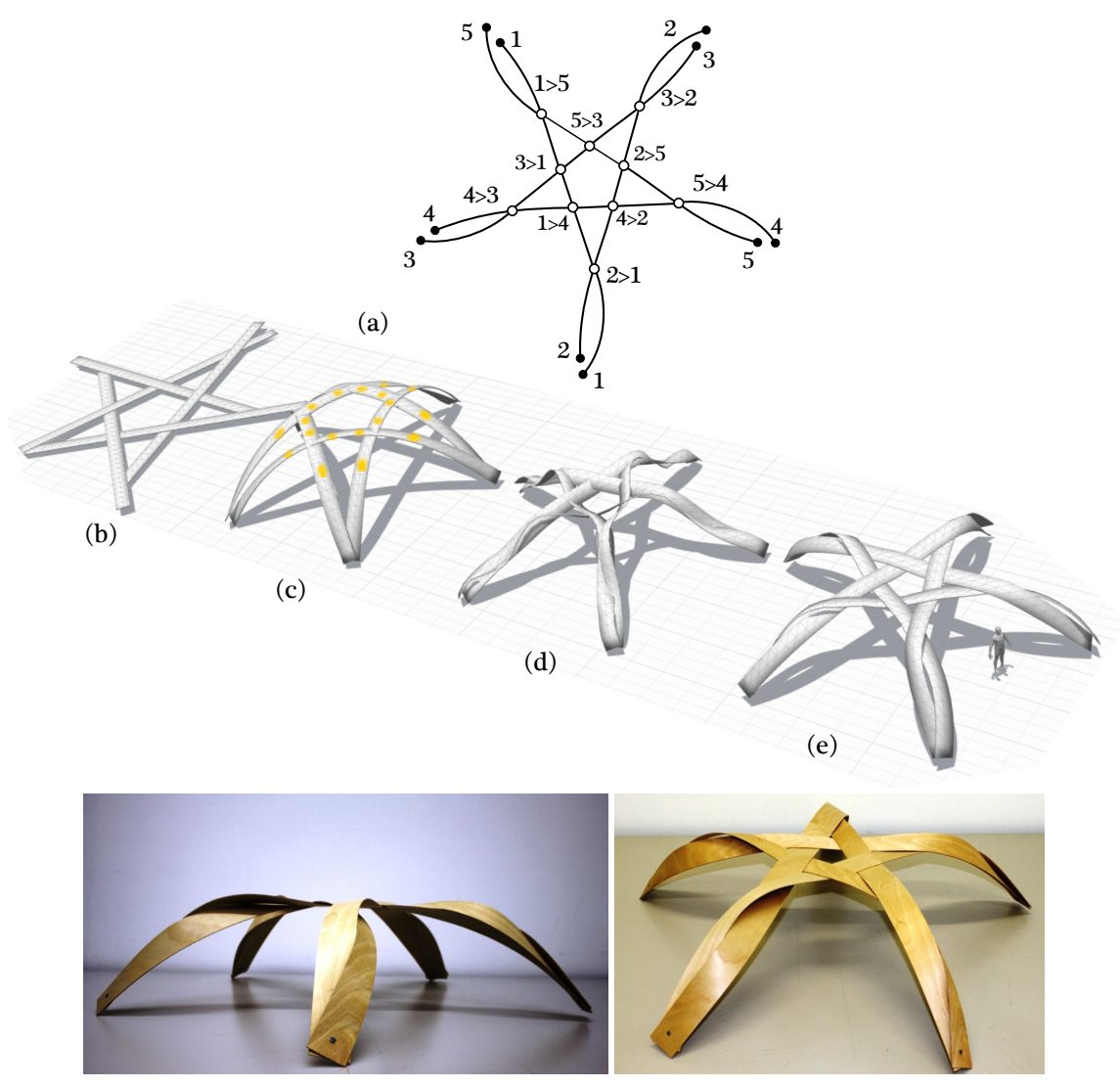

Figure 8: A pentafoil interlaced roof: (a) the graph representation (b) flat initial state (c) solution to the Twisted Elastica problem with overlapping vertices highlighted to be interlaced at next step (d) interlaced configuration (contains intersection) (e) intersections handled

beams) is $0.7 \mathrm{~m}$, the middle proximity constraint is set also to $0.7 \mathrm{~m}$ and the two top proximity constraints are $0.4 \mathrm{~m}$. (constrained vertices are highlighted in Fig. 9.c) All panels are all $0.2 m \times 0.01 \mathrm{~m}$. In concept structure Fig 9.d, the rebars are positioned to represent the imposed proximity while solving the constrained system and make the entire module behave as an active prestressed truss beam.

\section{Conclusion and outlook}

We borrowed two elegant models and techniques employed for physically based simulation of flexible bodies from computer graphics to use in structural formfinding and combined them as a simulation pipeline with a particular focus to ex- 


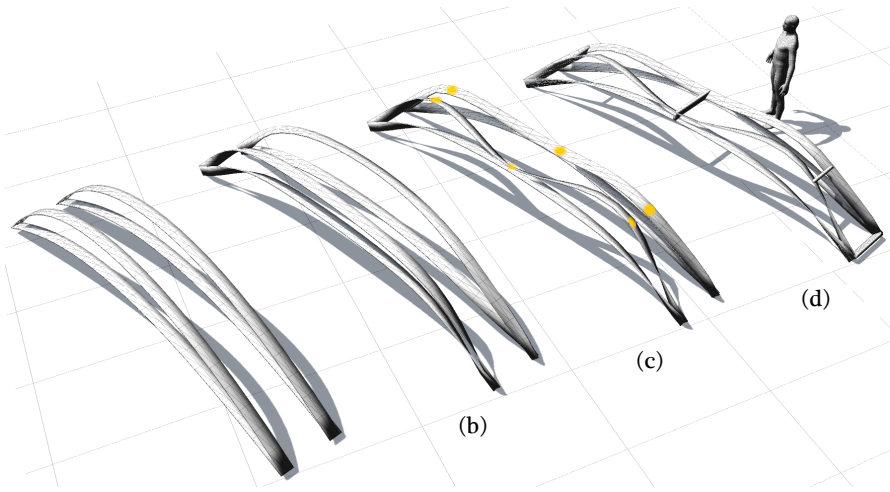

(a)
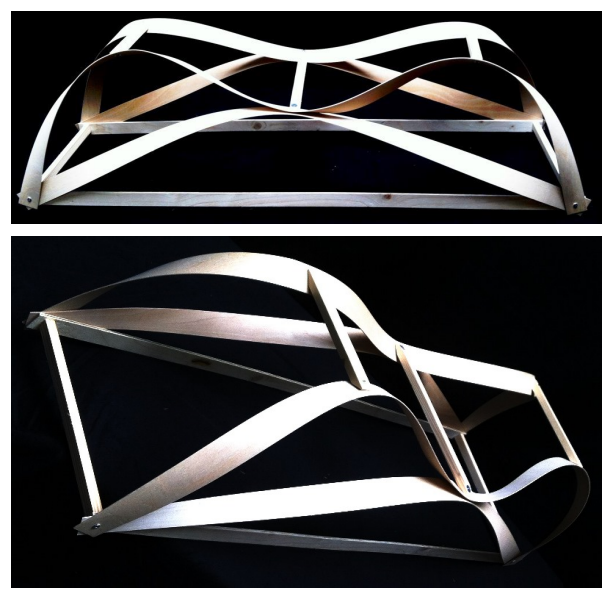

Figure 9: A twisted truss beam active module: (a) The solution for Elastica problem (b) twisted non-coupled beams (c) coupled system of twisted Elasticas with constrained vertices highlighted (d) a concept structure with connectors maintaining the enforced constraints

plore interlaced structures and modules and twisted configurations currently out of reach of non-engineers. The framework is subdivided into optimization subproblems to handle the Elastica problem in its zero-twist and twisted form and also in case of coupled system of panels with or without overlap constraints. Intersecting panels are cleared using a pseudo-dynamic shell model and an intersection contour minimization algorithm. Both rod and shell frameworks have been implemented as Grasshopper plug-ins and are concerted as described to run presented case studies. 


\section{Acknowledgments}

This work was sponsored by the Swiss National Science Foundation (SNSF) under grant No. 200021_137884/1. This support is gratefully acknowledged. Authors would like also to thank Basile Audoly and Eitan Grinspun for their constructive remarks on problem formulation and implementation.

\section{References}

BarafF, D., Witkin, A., And Kass, M. 2003. Untangling cloth. ACM Trans. Graph. 22, 3 (July), 862-870.

Bergou, M., Wardetzky, M., Harmon, D., Zorin, D., And Grinspun, E. 2006. Discrete quadratic curvature energies. In ACM SIGGRAPH 2006 Courses, ACM, 20-29.

Bergou, M., Wardetzky, M., Robinson, S., Audoly, B., And Grinspun, E. 2008. Discrete elastic rods. ACM Transactions on Graphics (TOG) 27, 3, 63 .

Bridson, R., Marino, S., AND FEdKiw, R. 2003. Simulation of clothing with folds and wrinkles. In Proceedings of the 2003 ACM SIGGRAPH/Eurographics symposium on Computer animation, Eurographics Association, 28-36.

Chentanez, N., Alterovitz, R., Ritchie, D., Cho, L., Hauser, K. K., GoldberG, K., Shewchuk, J. R., And O’Brien, J. F. 2009. Interactive simulation of surgical needle insertion and steering, vol. 28. ACM.

CHOI, K.-J., AND Ko, H.-S. 2005. Stable but responsive cloth. In ACM SIGGRAPH 2005 Courses, ACM, 1.

Dias, M. A., AND Audoly, B. 2014. A non-linear rod model for folded elastic strips. Journal of the Mechanics and Physics of Solids 62, 57-80.

Fleischmann, M., AND Menges, A. 2012. Icd/itke research pavilion: A case study of multi-disciplinary collaborative computational design. In Computational Design Modelling. Springer, 239-248.

Grinspun, E., Hirani, A. N., Desbrun, M., And Schröder, P. 2003. Discrete shells. In Proceedings of the 2003 ACM SIGGRAPH/Eurographics symposium on Computer animation, Eurographics Association, 62-67.

HAIRER, E., Lubich, C., AND WANNER, G. 2006. Geometric numerical integration: structure-preserving algorithms for ordinary differential equations, vol. 31. Springer.

HENSEL, M. 2013. Performance-oriented architecture: rethinking architectural design and the built environment. John Wiley \& Sons.

Hsl(2013). a collection of fortran codes for large scale scientific computation. http://www.hsl.rl.ac.uk.

HUDERT, M. 2012. Structural timber fabric: applying textile principals in building scale. $\mathrm{PhD}$ thesis, IBOIS, EPFL. 
Hughes, T. J., Pister, K. S., And TaYlor, R. L. 1979. Implicit-explicit finite elements in nonlinear transient analysis. Computer Methods in Applied Mechanics and Engineering 17, 159-182.

Lock, J., Laing, G., Mahvash, M., And Dupont, P. E. 2010. Quasistatic modeling of concentric tube robots with external loads. In Intelligent Robots and Systems (IROS), 2010 IEEE/RSJ International Conference on, IEEE, 23252332.

Love, A. E. H. 2013. A treatise on the mathematical theory of elasticity. Cambridge University Press.

Lu, J., AND ZHENG, C. 2014. Dynamic cloth simulation by isogeometric analysis. Computer Methods in Applied Mechanics and Engineering 268, 475-493.

Miller, J., Lazarus, A., Audoly, B., And Reis, P. 2014. Shapes of a suspended curly hair. Physical review letters 112, 6, 068103.

MÖLLER, T. 1997. A fast triangle-triangle intersection test. Journal of graphics tools 2, 2, 25-30.

Nitranen, J. 2013. kierretty ja kaareva: Warped and twisted. Puu - WoodHolz - Bois, 1, 22-27.

Olson, S. D., Lim, S., AND CorteZ, R. 2013. Modeling the dynamics of an elastic rod with intrinsic curvature and twist using a regularized stokes formulation. Journal of Computational Physics 238, 169-187.

PIKER, D. 2013. Kangaroo: Form finding with computational physics. Architectural Design 83, 2, 136-137.

Volino, P., AND MAgnenat-Thalmann, N. 2006. Resolving surface collisions through intersection contour minimization. In ACM Transactions on Graphics (TOG), vol. 25, ACM, 1154-1159.

WëChTER, A., AND BIEGLER, L. T. 2006. On the implementation of an interiorpoint filter line-search algorithm for large-scale nonlinear programming. Mathematical programming 106, 1, 25-57. 